\title{
Investigation of Leukemogenic Mechanisms using Metabolomic Approaches ${ }^{\dagger}$
}

\author{
Kamisetty Venkata Pranava Kavya ${ }^{1, *}$, Daniel Alex Anand 1,* \\ 1 Department of Bioinformatics and The Centre for Molecular Data Science and Systems Biology, Sathyabama Institute of \\ Science and Technology, Chennai - 600119, South India \\ * Correspondence: kavya.kavi1209@gmail.com; \\ $\dagger$ Presented at International e-Conference on Bioengineering for Health and Environment (ICBHE 2020)
}

Received: 5.07.2020; Revised: 10.07.2020; Accepted: 12.07.2020; Published: 15.07.2020

\begin{abstract}
Leukemogenic mechanisms are characterized by the rapid proliferation of abnormal white blood cells that agglomerate in the bone marrow and subsequently interfere with the making of the normal blood cells. AML is a disparate neoplastic disorder that typified by naïve myeloid cell upsurge and bone marrow incompetence. AML is the most familiar type of leukemia affecting the aged. One prognostic feature associated with this response is the predominance of triggering mutations in FMSlike tyrosine kinase 3 (FLT3), especially the eventuality of internal tandem duplication (FLT3-ITD). AML with FLT3-ITD mutation is demonstrated to have a poor prognosis. Metabolomic profiling is a tool for understanding the modalities of the metabolites. In this study, we have attempted to understand AML with FLT3 from a metabolomic perspective. We have identified all the metabolites and their participating pathways, which are implicated in Acute myeloid leukemia (AML). AML is a hematological perturbation resulting from the proliferation and intensification of malignant myeloid cells. Formerly, smaller numbers of metabolites have been of use in the diagnosis of complex metabolic maladies and monogenic disorders like the inborn errors of metabolism. The results of this study enabled us to generate a metabolomic profile of FLT3/ITD patients, and we further analyzed the pathways from a network biology standpoint in which these metabolites are vital. We analyzed molecular networks with the help of Cytoscape and its plugins like Metscape. Arachidonic acid and Purine metabolism in cellular metabolites, while Glycolysis and Gluconeogenesis pathways, urea cycle and metabolism of arginine, proline, glutamate, aspartate, and asparagine metabolisms in plasma metabolites were the pathways having the highest impact in AML FLT3/ITD.
\end{abstract}

Keywords: blood cells; leukemia; mutations; bone marrow; inborn errors of metabolism.

(C) 2020 by the authors. This article is an open-access article distributed under the terms and conditions of the Creative Commons Attribution (CC BY) license (https://creativecommons.org/licenses/by/4.0/).

\section{Funding}

This research received no external funding.

\section{Acknowledgments}

This research has no acknowledgment.

\section{Conflicts of Interest}

The authors declare no conflict of interest. 